\title{
To the question of the complexity of the social system
}

\begin{abstract}
Annotation
The article addresses the issue of the complexity of social systems. It is shown that the description of the activity of social systems is based in most cases on qualitative data. When considering mathematical models of complex social systems, it is proposed to eliminate complexity in social systems by applying the principles of similarity to the simplest subsystems of a social system.
\end{abstract}

Keywords: social system, model of social system, similarity, complexity, classification
Volume 8 Issue 4 - 2020

\author{
Alexey Lebedev \\ Professor of the Department of Economics, management \\ and applied Informatics, Institute of arts and information \\ technologies, Russia

\begin{abstract}
Correspondence: Alexey Lebedev, Professor of the Department of Economics, management and applied Informatics, Institute of arts and information technologies (branch of the Saint Petersburg Humanitarian University of trade unions), Doctor of technical Sciences, Institute of arts and information technologies, Russia, Tel +7-960-7058967, Email lebedevavic@rambler.ru
\end{abstract}

Received: June 22, 2020 | Published: August 3I, 2020

\section{Review}

The question of the complexity of the social system remains relevant to date. First of all, the complexity of a social system is determined by its dimension, when hundreds of thousands of elements, each of which is characterized by several tens of parameters, are part of the federal level social system. ${ }^{1}$ However, the use of modern DBMS to collect information about the activities of complex social systems solves the problem of its dimensionality.

Note that quite often the terms "large system" and "complex system" are used as synonyms. At the same time, some authors associate the concept of "large" with the number of elements in it, and the concept of "complex" with the complexity of the relationships, algorithms existing in the system, or the complexity of behavior. In this case, the use of modern DBMS only partially removes the problem of relations in the system, which can be regulated by the corresponding logical conditions, but the complexity of the algorithmic behavior can be eliminated only by building the corresponding mathematical model.

One way to consider the complexity of the system is to estimate the number of elements included in the system. ${ }^{2}$ For example, a quantitative assessment of the complexity of a system can be made by comparing the number of system elements (n) and the number of bonds $(\mathrm{m})$ using the following formula:

$$
S=\frac{m}{n(n-1)}
$$

where $n(n-1)$ is the maximum number of connections between elements of the system.

Based on the information approach to the description of the system, when the information subsystem of the social system is singled out in a separate way, it can be assumed that the structural complexity of the system should be proportional to the amount of information needed to describe it (remove uncertainty). In this case, the total amount of information about the system $\mathrm{S}$ in which the a priori probability of the appearance of the ith property is $\mathrm{p}\left(\mathrm{s}_{\mathrm{i}}\right)$ is defined as

$$
I_{s}=-\Sigma_{i} p\left(s_{i}\right) \ln p\left(s_{i}\right) .
$$

Thus, the complexity in the behavior of a social system can be eliminated only by constructing an appropriate mathematical model based on detailed data organized properly using modern DBMSs.

Mathematical modeling of the behavior of a social system is based on the assumption that systems that are homomorphically (isomorphically) represented as information models of varying degrees of detail (a descriptive description of the system), having similarities in the structure and work processes in the system, make it possible to summarize other types of similar social systems. Thus, the behavior of the system, as well as the processes occurring in it, are transferred to other systems similar to it, even if they are in other external conditions and have different structures and their own goals.

As a rule, mathematical modeling goes through a number of stages, the main of which is the selection of the most significant characteristics or sides of a real system, for which a mathematical model is built (the stage of image formation). For a rigorous explanation of the essence of replacing one object (real system) with another (image), we use the mathematical concepts of "homomorphism" and "isomorphism".

Recall that a set A with property (relation $G$ ) defined for its elements $\mathrm{x}_{\mathrm{i}}$ and a set $\mathrm{B}\left(\mathrm{y}_{\mathrm{j}} \in \mathrm{B}\right)$ with relation $\mathrm{R}$ are isomorphic if there is a one-to-one correspondence between them such that $\forall \mathrm{x}_{\mathrm{i}} \in \mathrm{A}$ (and relation $\mathrm{G}$ ) corresponds to one and only one element $\mathrm{y}_{\mathrm{i}} \in \mathrm{B}$ (with relation $\mathrm{R}$ ) and vice versa $\forall \mathrm{y}_{\mathrm{j}} \in \mathrm{B}$ corresponds to one and only one element $\mathrm{x}_{\mathrm{i}} \in \mathrm{A}$.

From the point of view of modeling, this means that there is no difference between the two systems (objects), each of them can be considered as a model of the other. 
The set $B$ with the relation $R$ defined for its elements $y_{j}$ is a homomorphic image of the set A: $\left(x_{i} \in A, G\right)$ if: 1) $\forall x_{i} \in A$ corresponds to one and only one element $\mathrm{y}_{\mathrm{j}} \in \mathrm{B}$, and the converse is not true; 2) the fact that $\forall \mathrm{x}_{\mathrm{i}} \in \mathrm{A}$ has property $\mathrm{G}$ implies that the corresponding element $y_{j} \in B$ has property $R$ and vice versa.

From these definitions it is clear that the mathematical model of a social system serves a homomorphic image of a real system and, in turn, is isomorphic to the image that is obtained from a real system by extracting essential characteristics from it. Thus, a real system can have several different homomorphic images or models. In practice, this is what happens. Each mathematical model describes not the whole object, but its individual parts, the sides most significant from the point of view of the research tasks.

In our opinion, overcoming the complexity of algorithms or the complexity of the behavior of social systems is possible only on the basis of consideration of information processes taking place in the social system. At the same time, the information subsystem of the social system includes the set of Q resources necessary for decisionmaking, the set $(\mathrm{Y}, \mathrm{Z})$, where $\mathrm{Y}$ is the set of "controlled" initial conditions or inputs; $\mathrm{Z}$ a lot of "uncontrollable" initial conditions or inputs. That is why, the main efforts in the preparation and adoption of managerial decisions should be focused on increasing the level of organization of the processes of collecting, processing and mandatory procedures for analyzing information.

It should be noted that the large amounts of text data accumulated to date in social systems are already an information resource. When creating them, in most cases, a declarative approach is used that is aimed at creating a repository of information of such a structure in which statements, axioms, previously developed rules for finding solutions, etc. can be placed. The description of the social system is built in most cases on a verbal basis, but if the description is based on a system of indicators, then for most even similar social systems these indicators are very diverse and not comparable. Note that the criteria for the similarity of social systems have not been formulated until recently, and it is not possible to draw an unambiguous conclusion about their similarity.

The accuracy of the available data and the frequency of updating information in various (including similar) systems also differ significantly from each other. The influence of external conditions at different time periods on the behavior of the system is also not considered in most cases; in the best case, a certain correction factor is introduced. All this extremely negatively affects the application of the principles of the general theory of systems to the description of the structure and processes taking place in social systems.

Social complexity arises when solving problems at all levels of management from local to global, that is, from the lowest (primary) level of management to the highest level. In most cases, in modern conditions, the difficulty in solving such problems by a leader is reduced exclusively to an economic function. Therefore, it is believed that the social system in brow is similar to its economic subsystem for which the corresponding mathematical model is being built. In most cases this is not true.

This is due to the fact that control over the activities of the social system is, first of all, carried out precisely through the line of spending funds, and for all the rest, if carried out, then according to the residual principle (for example, remember when the control was last carried out in your institution socio-psychological climate and when control of financial discipline). However, social systems also have a social function for which the economic component is largely secondary.

In addition, most managers are used to being guided by linear one or two parametric mathematical models, the construction of which, in most cases, is just carried out for economic data. And for social systems, characteristic non-linear mathematical models are typical. ${ }^{2}$

\section{When considering the complexity of the social system, there are} several problematic issues. Where does this difficulty come from?

Firstly, it is the verbality of the available descriptions of most social systems and the qualitative data used in their description. Note that, unfortunately, we communicate not in the language of dependencies and mathematical models, but emotionally, characterizing the current situation mainly fuzzy linguistic parameters. The parameters used in communication can be ranked by using an ordinal or nominal scale of 3-5 signs, which will somewhat simplify the construction of a mathematical model to describe the social system, but even in this case we will be far from the usual interval of signs.

Secondly, the slow development of social processes (to a certain point), which for the time being are not perceived as a problem (for example, the state of pension reform in Russia). Thirdly, the absence of some critical values of indicators of social problems, instead of which there is a subjective opinion of the leader (yesterday it was still tolerable, and today the situation is close to collapse). ${ }^{3}$

Mathematical models are traditionally used in the study of dynamic processes or objects and represent their description, made in mathematical form. From the point of view of formal mathematical notation (symbols, relationships, etc.), these models are not much different from the mathematical models used in research in other areas. At the same time, the separation of mathematical models describing the activities of social systems into a separate subclass is quite justified and is a consequence of the really existing qualitative differences, expressed, including in mathematical form. In other words, mathematical models of social systems are a set of scientific disciplines that lie at the intersection of statistics, linguistics and mathematics, the task of which is to simulate the processes occurring in social systems using mathematics.

Our analysis of the most used mathematical methods allowed us to combine them into three main groups in the following areas; ${ }^{4}$ statistical methods related to the analysis and processing of statistical data related to the activities of social systems; forecasting - methods designed to test mathematical models of social systems based on the results of observations based on available statistics; methods leading to finding optimal solutions.

The use of certain methods of researching a specific object is associated with the construction of certain mathematical models corresponding to this direction. For statistical methods, probabilistic models, regression and correlation analysis models, and factor models are most characteristic.

A wide range of mathematical methods and models describing the activities of social systems is due to the extreme complexity of real processes and systems. This complexity specifies the specifics of the application of the method of mathematical modeling in this area, the main idea of which correlates with the limitations regarding the possibilities of mathematical modeling of social processes. 
If we turn to the biological theory of the complexity of systems, then it is based on the provision on the excess energy entering the system, as well as the presence of competition between biological species that stimulate either speciation or lead to outgoing flows from systems of this kind.

Arguing in a similar way, it can be assumed that the presence of excess resources in the social system ${ }^{5,6}$ creates greater diversity in its structure and organization, which in turn, by analogy with biological systems, should increase the stability of the social system. This conclusion, built on an analogy, seems very naive to us. The thing is that in the social system, in addition to the phenomena of self-organization characteristic of biological systems, there is also a management of structure and processes. And the management of complex systems, both in structure and in the processes that occur in them, is an extremely difficult task, which often translates only into the economic plane, where process management is somewhat simpler. But at the same time, successful solution of the tasks of the economic subsystem may not lead to the achievement of the general goal of the system.

In a social system, the ratio of complexity to stability or sustainability is much more diverse than in biological systems, where diversity is equated with stability. The complexity of social systems often generates increased resource consumption, and vice versa, excess resources generate complexity. In many respects, the successful development of social systems is connected precisely with the excess of available resources, but at the same time, the difficulty in social systems arises from the inability (or impossibility) to properly manage these resources.

Thus, the stability of a social system is determined by the complexity of its composition and the processes taking place in it. But I would like to directly identify a number of factors affecting the stability of the social system. In a social system, complexity is fundamentally linked to sustainability, but this relationship is not simple, not linear, and not constant.

It is obvious that social systems are sufficiently stable over a wide range of time, and the collapse of the social system itself can occur only if the level of available material resources falls below the ability to provide priority needs and this situation will continue for more than two years. Such situations for most social systems can only be caused by a significant change in external conditions.

If we talk about the possibility of abstract formalization, then mathematical models of the activity of social systems can be represented as a system of equal flows of costs and benefits, which reflect the degree to which citizens realize the benefits of combining their individual and public interests, depending on the degree of consciousness, culture, qualifications, etc. The most characteristic of these dependencies are for small subsystems of the social system. Note that for systems of this kind, data are subject to direct verification in the process of control checks; moreover, these data are not so large in dimension and, moreover, are quite reliable. Note that the qualitative characteristics of social systems are still not satisfactorily expressed in quantitative terms. As noted above, all significant social processes have the same focus - from the emergence of needs to their satisfaction.

Thus, if we summarize all of the above, then the concept of mathematical modeling of social systems based on qualitative data is based on the following basic principles: equivalence of the total costs of society for social systems and utility derived from their activities; focus on innovation, i.e. prospective needs of the individual;

self-organization of social systems in the direction of increasing social efficiency.

The internal goal of social systems is, to a greater extent, the stability or preservation of the invariability of the current situation. These provisions are not characteristic of a narrow social subgroup of persons aged 17-24 years. It should be noted that due to the narrow time interval, this age group is not predominant in composition in most social systems. Most of us prefer existing comfort (stability) over an unusual way of life, or some extreme changes (fault tolerance). However, a fully sustainable society would be worthless in terms of development. We emphasize once again that in this case we are talking about the goals of the system itself, that is, most of the elements of its components.

If we talk about the general goal of the social system, then we are talking about fault tolerance, that is, the continuity of political values that underlie statehood. The relationship of the external environment with the stability of the social system is indirect and subtle. The stability of the social system is not the achievement of stagnation (it seemed that in the 80 s of the 20th century the USSR was completely stable, but in a fairly short time the system came to a social collapse). Sustainability should be understood as the gradual satisfaction of the growing needs of members of the social system, for which the state system of the Russian Federation was not ready for the period of the late 20 th century.

In recent years, there has been a tendency for all types of resources to grow in social systems, this is a population growth; differentiation and specialization in social roles of representatives of social systems; increase the production of information.

We are increasingly confronted with problems and react to them by creating new management institutions, adding more specialists or bureaucratic levels to the institution, collecting and processing additional information. Although most managers prefer not to complicate management procedures in the organization, emerging issues can be sources of complexity.

This principle can be illustrated in two main areas of problem solving: resource creation and information production. Until recently, society has taken the path of increasing material resources; the more resources are produced, the more efficient the organization is. Until today, the growth of gross domestic product (GDP) is one of the main characteristics of the development of the state. However, how effective is the management of these generated resources?

The amount of information circulating in social systems is still insufficient for unambiguous decision making. Until the uncertainty in the data is minimized due to the increase in the volume of processed data, the situation will not change. Given the uncertainty in the source data, the decision-making process in social systems, as a rule, takes place creatively, that is, in a non-formalized way. The existing cases of formalization of management decisions are not recorded and not systematized, the decision-making process is largely based on verbal and textual information, and only in very rare cases are mathematical methods successfully applied. In contrast to the above, the existing formal procedures for the preparation and adoption of managerial decisions are based on a wide array of numerical data, ${ }^{5}$ text data for this is maximally formalized or excluded from the algorithms. 
In order to make managerial decisions correctly, a specialist must perform a detailed analysis and forecasting the development of a specific situation, apply the requirements of guidance documents in the current conditions, gain experience in choosing decisions, develop action plans, etc. As any society increases its complexity, it becomes more and more dependent on information, and members of the social system require a higher level of education. ${ }^{6}$

Social systems are always problematic systems, and the main problem they are facing is managing them. Solving problems, according to most modern managers ("effective managers"), is an economic process for which they assume that there are benefits. Efforts to solve problems, as can be seen from the examples of creating resources and obtaining information, usually develop along the path of increasing complexity. Ultimately, problem-solving efforts can become more complex, costly, and ineffective, as they either end, or collapse, or require large subsidies.

However, as a rule, when the solution to the problem is completed, this is a fairly short-term measure. Damage that may arise in the future is associated with cumulative and long-term effects that are not usually worked out.

In a social system with a hierarchical type of management, the benefits of increasing complexity arise and are used at the upper level of management, while the costs of this process increase mainly at the lower level of the system. These costs are usually absolutely not taken into account when developing solutions. This leads to the fact that if you follow all the instructions of a higher system, then lower level employees simply do not have enough time to execute them, as a result, they simply ignore some of the instructions that are less important from their point of view (and their point of view is not always true) Ignoring part of the instructions of the superior system leads to the fact that out of habit, the critical instructions of the superior system also begin to be ignored.?

In such a general situation, the costs and benefits of complexity are not related to each other. In a social system with a hierarchical type of control, this means that information about the cost of complexity must pass extremely accurately and quickly. However, it is precisely for social systems with a hierarchical type of control that the flow of information going from bottom to top, often inaccurate and ineffective, is characteristic, since the data is determined by the requirements of a higher system, which either does not take into account or weakly takes into account the situation at the lower levels of management. In fact, all solutions to the problem of the complexity of social systems are temporary. As modern social systems generate ever greater complexity in their structure, there is no optimal level of complexity that should be monitored and maintained as a result of observation of the social system.

\section{Acknowledgments}

None.

\section{Conflicts of interest}

The author declares there are no conflicts of interest.

\section{References}

1. Ovchinsky AS, Lebedev AV, Irikov VA. The current state of information technology management decision-making in social systems. Proceedings of the Academy of Management of the Ministry of Internal Affairs of Russia. 2017;4(44):195-199.

2. Lebedev A, Shmonin A, Vasiliev F, et al. Principles of mathematical models constructing based on the text of qualitative data of social systems. Studies in system, decision and control. 2019;181:29-38.

3. Lebedev AV. A mathematical model of the controlled process of creating requirements for the adaptive state of a social system. Economics and Management Systems Management. 2017;26(4-3):372-378.

4. Lebedev AV. Information support of the decision-making process in the Federal Penitentiary Service of Russia. Bulletin of the Vladimir Law Institute of the Federal Penitentiary Service of Russia. 2011;3(20):3845 .

5. Hornik K, Stinchcombe M, White H. Universal approximations of an unknown mapping and its derivatives using multilayer feed forward networks. Neural Networks. 1990;3(5):551-560.

6. Lebedev AV. A mathematical model for assessing the resource support of a social system. Tomsk State University Journal. 2018;1(46):51-55.

7. Lebedev AV. On finding the analytical form of the crime recovery function. Man: crime and punishment. 2013;3(82):108-112. 